ARTICLE

\title{
Enhanced performance of in-plane transition metal dichalcogenides monolayers by configuring local atomic structures
}

Yao Zhou (1) ${ }^{1,8}$, Jing Zhang (10 2,8, Erhong Song ${ }^{3,8}$, Junhao Lin ${ }^{4,8}$, Jiadong Zhou ${ }^{5}$, Kazu Suenaga (i) ${ }^{6}$, Wu Zhou ${ }^{7}$, Zheng Liu (i) ${ }^{5}$, Jianjun Liu (i) ${ }^{3 凶}$, Jun Lou ${ }^{2 \otimes} \&$ Hong Jin Fan (10 ${ }^{1 凶}$

The intrinsic activity of in-plane chalcogen atoms plays a significant role in the catalytic performance of transition metal dichalcogenides (TMDs). A rational modulation of the local configurations is essential to activating the in-plane chalcogen atoms but restricted by the high energy barrier to break the in-plane TM-X $(X=$ chalcogen) bonds. Here, we theoretically design and experimentally realize the tuning of local configurations. The electron transfer capacity of local configurations is used to screen suitable TMDs materials for hydrogen evolution reaction (HER). Among various configurations, the triangular-shape cobalt atom cluster with a central sulfur vacancy $\left(3 \mathrm{CO}_{\mathrm{Mo}}-V_{S}\right)$ renders the distinct electrocatalytic performance of $\mathrm{MoS}_{2}$ with much reduced overpotential and Tafel slope. The present study sheds light on deeper understanding of atomic-scale local configuration in TMDs and a methodology to boost the intrinsic activity of chalcogen atoms.

\footnotetext{
${ }^{1}$ School of Physical and Mathematical Sciences, Nanyang Technological University, Singapore 637371, Singapore. ${ }^{2}$ Department of Materials Science and Nano Engineering, Rice University, Houston, TX 77005, USA. ${ }^{3}$ Shanghai Institute of Ceramics, Chinese Academy of Sciences, 200050 Shanghai, China. ${ }^{4}$ Shenzhen Key Laboratory of Advanced Quantum Functional Materials and Devices, Department of Physics, Southern University of Science and Technology, 518055 Shenzhen, China. ${ }^{5}$ School of Materials Science and Engineering, Nanyang Technological University, Singapore 639798, Singapore. ${ }^{6}$ National Institute of Advanced Industrial Science and Technology (AIST), Tsukuba 305-8565, Japan. ${ }^{7}$ School of Physical Sciences and CAS Key Laboratory of Vacuum Sciences, University of Chinese Academy of Sciences, 100049 Beijing, China. ${ }^{8}$ These authors contributed equally: Yao Zhou, Jing

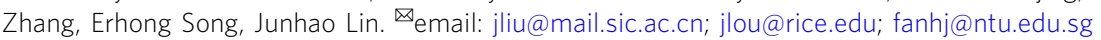


M $\mathrm{oS}_{2}$ is a promising candidate to replace Pt for electrocatalytic hydrogen evolution reaction (HER) due to its environmental friendly and low cost characteristics ${ }^{1-3}$. While increasing the conductivity via forming heterojunction bilayer with a conductive substrate can promote the overall catalytic performance ${ }^{4-6}$, the performance of pristine $\mathrm{MoS}_{2}$ is restricted by the density of active sites ${ }^{7-10}$. The pursuit to maximize $\mathrm{MoS}_{2}$ utility inspires researchers to explore various ways to rouse the activity of inert sulfurs in the $\mathrm{MoS}_{2}$ basal plane. For example, edge-site engineering ${ }^{9,11}$, phase transformation ${ }^{12,13}$, amorphization $^{14,15}$, and in-plane doping/vacancy modifications ${ }^{16-20}$ have been reported. Notably, changing the local configurations ${ }^{18,21-24}$ by introducing atomic defects (doping or vacancy) is preferable as defected $\mathrm{MoS}_{2}$ exhibits better stability compared to transformed $1 \mathrm{~T}^{\prime}$ phase ${ }^{25}$ and amorphous $\mathrm{MoS}_{2}{ }^{10}$. However, the reported activity of in-plane sulfur enhanced by local configuration modification is still far from that of Pt-based catalysts ${ }^{26}$. This is because the large energy are required to break the in-plane Mo-S bonds. In fact, few types of atomic local configurations have been realized in the basal plane of $\mathrm{MoS}_{2}{ }^{27-30}$, so the tuning ability of local configurations is quite limited so far ${ }^{16}$. Hence, in order to improve the intrinsic activity of in-plane sulfur atoms, it is essential to understand the intrinsic correlation and explore new methodologies to enrich stable and highly efficient local configurations.

Herein, we conducts both computational and experimental investigations in order to establish a correlation between local configuration and the electrocatalytic activity of monolayer $\mathrm{MoS}_{2}$. A group of stable local configurations with non-noble period-IV single atom or clusters ( $\mathrm{Co}, \mathrm{Fe}, \mathrm{V}$, and $\mathrm{Cr}$ ) accompanying additional sulfur vacancy in the in-plane domain of $\mathrm{MoS}_{2}$ have been attained. Given the correlation between binding strength and local configurations electronegativity, the activity of in-plane sulfur can be regulated by electron transfer capacity of local configurations. The peculiar triangular-shape Co atom cluster surrounding one sulfur vacancy configuration (viz., $3 \mathrm{Co}_{\mathrm{Mo}}-V_{S}$ ) is identified by both calculation and experiments to be most efficient to activate the inert sulfur sites. Correspondingly, a distinct enhancement in HER activity is achieved ( $\eta_{10}: 75 \mathrm{mV}$ and Tafel: $\left.57 \mathrm{mV} \mathrm{dec}^{-1}\right)$, exhibiting the highest intrinsic HER activity among $\mathrm{MoS}_{2}$ materials. The microcell HER measurements show a volcano-like relationship between content of specific local configuration and activity, which corroborates the optimized concentration of $3 \mathrm{Co}_{\mathrm{Mo}}-V_{S}$. Therefore, as demonstrated in the present work, it is possible to further activate the in-plane sulfur sites by rational engineering of the local configurations. This result may provide a route to unleash the electrocatalytic potential of TMD materials for efficient hydrogen generation in acidic solutions.

\section{Results}

Design efficient and stable local configurations. The activation of the basal plane in TMDs have been extensively studied to achieve the stable structure and enhance their catalytic activity ${ }^{31-33}$. Sulfur vacancy $(V s)$ on the surface is an electron donor and can induce a localized gap state in $\mathrm{MoS}_{2}$. Below a critical carrier density, the transport of donor states is governed by nearest-neighbor hopping at high temperatures and variable-range hopping (VRH) at low temperatures ${ }^{23,34-36}$. Regional charge states around a defect structure are suggested to make an important contribution to regulating the catalytic activity. Based on the above analysis, we believe that it is reasonable to monitor the defects induced Bader charge fluctuation, and the $\mathrm{H}$ adsorption to define the active sites through DFT computational screening.

To study the TM and synergistic effect of $V s$ on sulfur sites, we have considered six configurations including TM atoms (TM: Co,
$\mathrm{V}, \mathrm{Fe}$, and Cr; TM amounts from 1 to 3 ) with or without $V s$ (structures see in Supplementary Fig. 1 and Supplementary Note 1) that are set as models to screen stable catalytic structures through DFT calculations. The hydrogen adsorption free energy $\left(\Delta G_{\mathrm{H}}\right)$ is an effective descriptor to predict the activity for various catalyst systems ${ }^{37}$. The ideal value of $\Delta G_{\mathrm{H}}$ is $0 \mathrm{eV}$, which corresponds to a thermoneutral state of the adsorbed atomic hydrogen and efficient proton/electron transfer and hydrogen release ${ }^{1}$. The correspondingly calculated $\Delta G_{\mathrm{H}}$ of monolayer $\mathrm{MoS}_{2}$ with varied local configurations are further exhibited in Supplementary Fig. 2 and Supplementary Table 1, indicating the stronger $\mathrm{H}^{*}$ adsorption on atomically structured $\mathrm{MoS}_{2}$ than on intact $\mathrm{MoS}_{2}$. In addition to intrinsic activity $\left(\Delta G_{\mathrm{H}}\right)$, structural stability affecting the final electrochemical durability of catalysts should be considered. Based on the formation energies of all possible configurations (Supplementary Fig. 3), the $3 \mathrm{Co}_{\mathrm{Mo}}-V s$, $3 \mathrm{Fe}_{\mathrm{Mo}}-V s, 1 \mathrm{~V}_{\mathrm{Mo}}$ and $1 \mathrm{Cr}_{\mathrm{Mo}}$ are identified as the most stable structures in the different possible TM-introduced $\mathrm{MoS}_{2}$ (Fig. 1a). Together with the activity (value of $\Delta G_{\mathrm{H}}$ ), the $3 \mathrm{Co}_{\mathrm{Mo}}-V s$ is expected to be the potential structure with both good stability and high activity. The predicted HER activity of $\mathrm{MoS}_{2}$ with different local configurations following the trend $3 \mathrm{Co}_{\mathrm{Mo}}-V s>1 \mathrm{~V}_{\mathrm{Mo}}>$ $3 \mathrm{Fe}_{\mathrm{Mo}}-V s>1 \mathrm{Cr}_{\mathrm{Mo}}$ (Fig. 1b). This trend remains the same with solvation correction, as demonstrated by our calculations with the implicit solvation model (Supplementary Fig. 4). The hydrogen adsorption free energy $\left(\Delta G_{\mathrm{H}}\right)$ on basal plane of intact $\mathrm{MoS}_{2}$ is far away from the optimal value. After tuning by local configurations, the $\Delta G_{\mathrm{H}}$ value of $-0.085 \mathrm{eV}$ comparable to that of $\mathrm{Pt}^{38}$, is achieved due to the much stronger bonding strength in $\mathrm{S}$ atoms with the assistance of $3 \mathrm{Co}_{\mathrm{Mo}}-V s$, which surpasses predicted activity of edge sites ${ }^{8}$. As expected, different configurations induce varied activity; all the structures with co-existence of $V s$ and $\mathrm{TM}_{\mathrm{Mo}}$ atoms synergistically tunes the $\Delta G_{\mathrm{H}}$ when compared to single one (Supplementary Table 1). In addition, the monolayers are used instead of porous 3D materials, minimizing the double layer effect induced by porosity. It is supported by CV curves in non-Faradaic region (blue rectangular in Supplementary Fig. 5) with nearly no hysteresis loop.

It is important to reveal the underlying mechanism of enhanced catalytic activity due to the local configuration. The above analysis indicates that the defects (TM substitution and Svacancy) and $\mathrm{H}$ adsorption could induce charge fluctuation of the regional structure due to electron delocalization of $\mathrm{MoS}_{2}$. In principle, the catalytic activity depends on the charge transfer capacity before and after $\mathrm{H}$ adsorption. To identify the effective catalytic structure, we show the nearest and the next-neighbor atoms which possibly induce a charge fluctuation in HER (Supplementary Fig. 6 and Supplementary Note 2). First, the nearest metals (nMo and doped $(3-n) \mathrm{TM}, n=0,1,2)$ and the adsorption $\mathrm{S} 1$ atom have relatively large change in charge (Fig. 1d-f, Supplementary Figs. 7-10). As a result, we consider $(3-n)$ TM-S-nMo as the first-order catalytic structure that is comprised of TM substitutes, adsorption $S$ atom, and the nearest Mo atoms. In contrat, the change in charge for the next-neighbor $\mathrm{S}$ and Mo atoms is relatively small. So they are considered as the second-order structure as the distance from the adsorption site is large. As a result, it is reasonable to assume the charge regulation of the second-order catalytic structure has a negligible effect on that of the first-order one. The radial distributions of charge distribution are presented in Supplementary Figs. 7-10. Therefore, in our study, we calculate the total charge difference of adsorption $S$ atom and the nearest metals to depict the charge transfer capacity to S-H bonds. The amount of charge transfer of local configuration (namely, atoms to induce the charge transfer includes: nearest nMo, doped $(3-n) \mathrm{TM}, n=0,1,2$ and adsorption $\mathrm{S} 1$ atom) is linearly correlated with $\Delta G_{\mathrm{H}}$. This result 
a

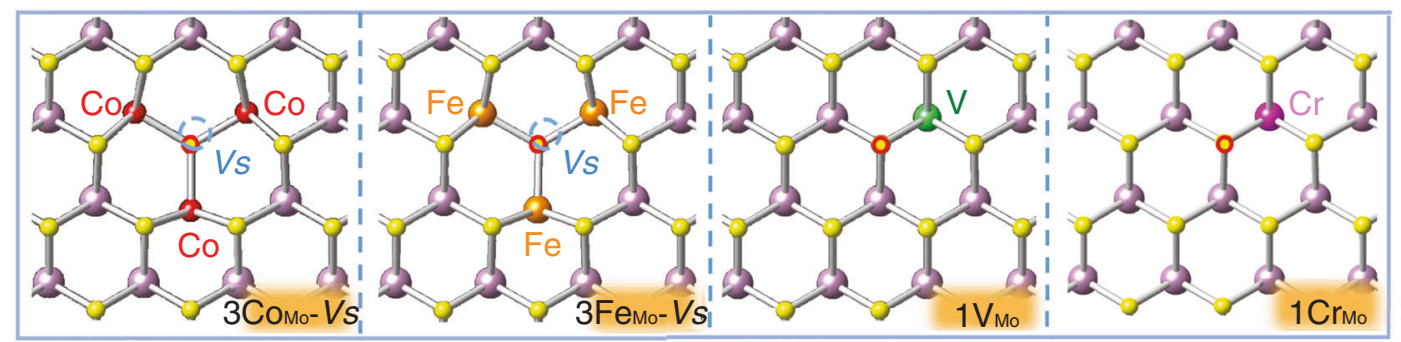

b

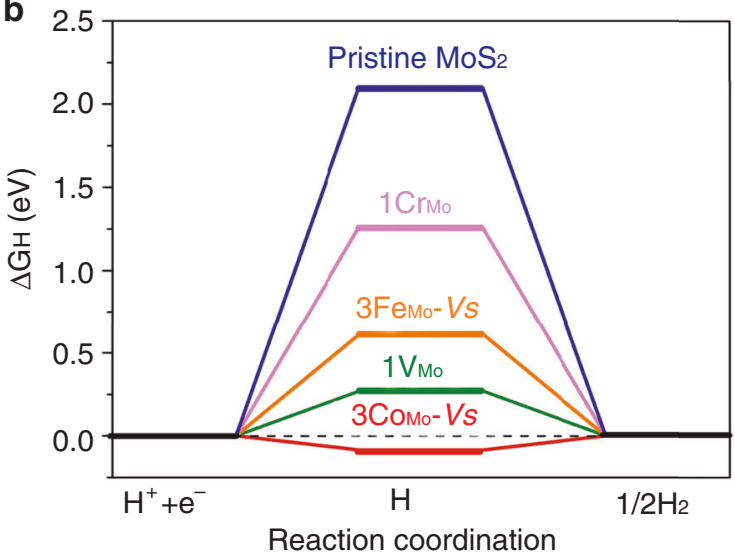

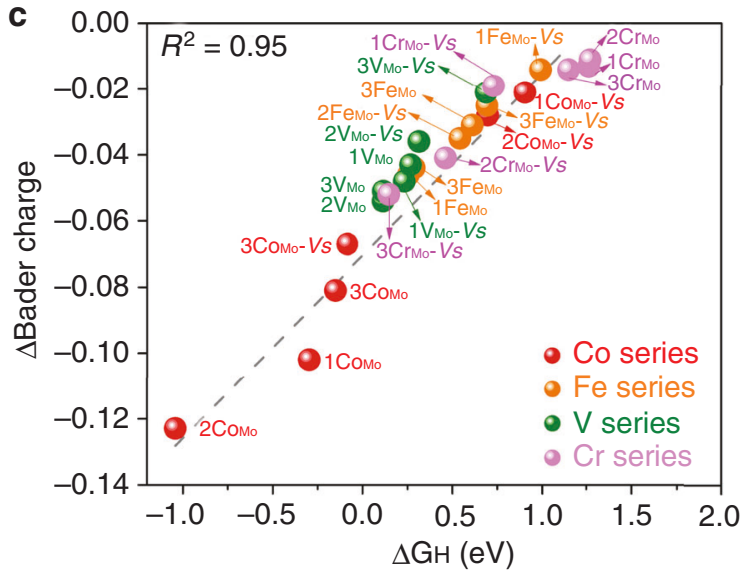
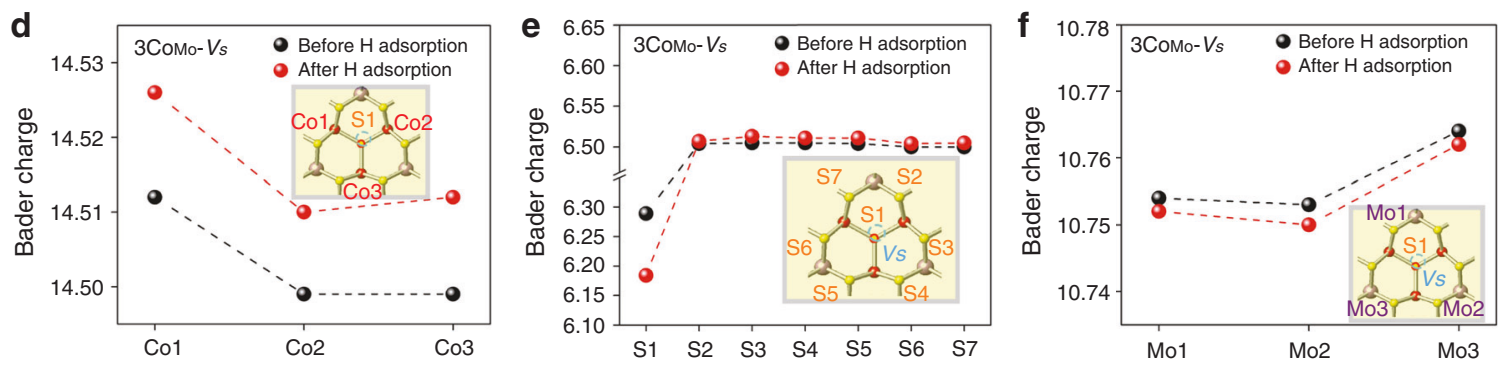

Fig. 1 DFT calculations to predict effect of local configurations to HER activity of $\mathbf{M o S}_{\mathbf{2}}$. a The most stable structures of $\mathrm{MoS}_{2}$ with $3 \mathrm{Co}_{\mathrm{Mo}}-V_{\mathrm{s}}$, $3 \mathrm{Fe}_{\mathrm{Mo}}-\mathrm{Vs}, 1 \mathrm{~V}_{\mathrm{Mo}}$, and $1 \mathrm{Cr}_{\mathrm{Mo}_{\mathrm{o}}}$ configurations and the $\mathrm{S}$ bonding with $\mathrm{H}$ is marked as red circles. $\mathbf{b}$ The free energy diagram of corresponding configurations and pristine $\mathrm{MoS}_{2}$. c The correlation between change of Bader charge of local configuration around sulfur atoms and hydrogen adsorption free energy $\left(\Delta G_{H}\right)$. The dashed line is linearly fitted with $R^{2}=0.95$. The Bader charge changes of $\mathbf{d}$ Co atoms, $\mathbf{e} 1$ and next-neighbor $\mathrm{S}$ atoms, and $\mathbf{f}$ the next-neighbor Mo atoms when $3 \mathrm{Co}_{\mathrm{Mo}}-\mathrm{Vs}$ is introduced in the $\mathrm{MoS}_{2}$.

indicates a charge regulation effect by the local configuration on HER activity (Fig. 1c). The linear correlation indicates that charge transfer capacity induced by varied local configurations are mainly delocalized in the first-order catalytic structure instead of on individual sulfur atoms. We found that, a charge difference around $0.07 e^{-}$(which corresponds to $\Delta G_{\mathrm{H}}=0 \mathrm{eV}$ ) should correspond to a high HER catalytic activity.

Realization and characterizations of local configurations. In light of the superior activity induced by the predicted local configurations, we employ the chemical vapor deposition (CVD) method to synthesize several monolayer $\mathrm{MoS}_{2}$ samples with various in-plane local configurations (Methods). The optical images of $\mathrm{Co}, \mathrm{Fe}, \mathrm{Cr}$, and $\mathrm{V}$-containing $\mathrm{MoS}_{2}$ monolayers are shown in Supplementary Fig. 11a-d. Raman spectra confirm that all the as-prepared samples preserve the lattice structure of $\mathrm{MoS}_{2}$ (Supplementary Fig. 12), as seen from the characteristic $A_{1 g}$ mode at $\sim 401 \mathrm{~cm}^{-1}$ and the $E_{2 g}{ }^{1}$ mode at $\sim 381 \mathrm{~cm}^{-1}$ observed in pristine $\mathrm{MoS}_{2}$ monolayer ${ }^{39}$. In addition, the Raman mappings indicate homogenous elemental distribution (Supplementary
Fig. 13). Atomic force microscopy (AFM) measurements further confirm that the as-prepared $\mathrm{MoS}_{2}$ domains are monolayers (Fig. 2a-d) with a thickness ranging between 0.7 and $0.9 \mathrm{~nm}$. As for the the system with small doping concentration, the peak-shift is ascribed to the dopant induced Fermi level movement ${ }^{40}$. However, the shifts of X-ray photoelectron spectra in both Mo 3d and $S 2 p$ are very small (below $0.3 \mathrm{eV}$, see Supplementary Fig. 14), likely due to the low dopant concentrations. Therefore the minor peak shift cannot justify if the dopants incorporate into the $\mathrm{MoS}_{2}$ lattice. More evidence is provided by the high-resolution spectra of TM 2p (Supplementary Fig. 15), which show clearly the formation of metal-sulfur bonds in all samples and support the substitutional dopants within the $\mathrm{MoS}_{2}$ lattice.

The annular dark-field (ADF) scanning transmission electron microscopy (STEM) imaging and electron energy loss spectroscopy (EELS) are used to further confirm the local atomic configurations. Figure $2 \mathrm{e}-\mathrm{h}$ show the atomic structure of the Co-, $\mathrm{Fe}-, \mathrm{V}-$, and $\mathrm{Cr}$-containing $\mathrm{MoS}_{2}$ monolayers, respectively. All four images show the lattice of $\mathrm{MoS}_{2}$ with Mo and $\mathrm{S}$ atoms alternating in bright and dim spots periodically. The TM atoms, which occupy the metal sites, show lower image contrast than 
a
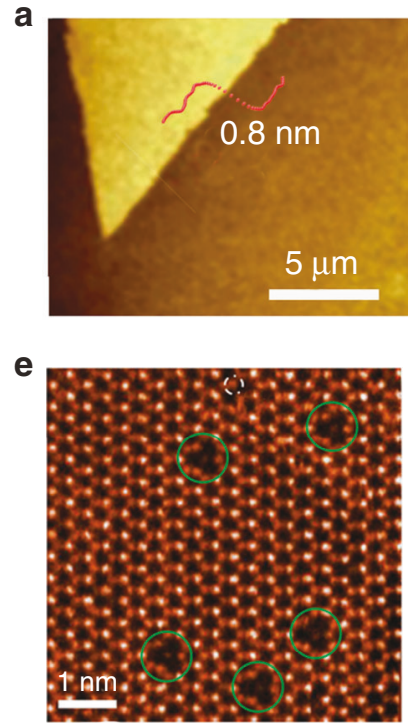

i

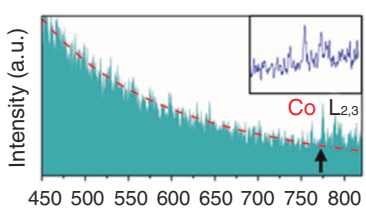

Energy loss (eV)

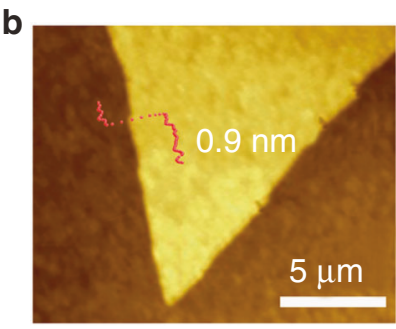

$f$
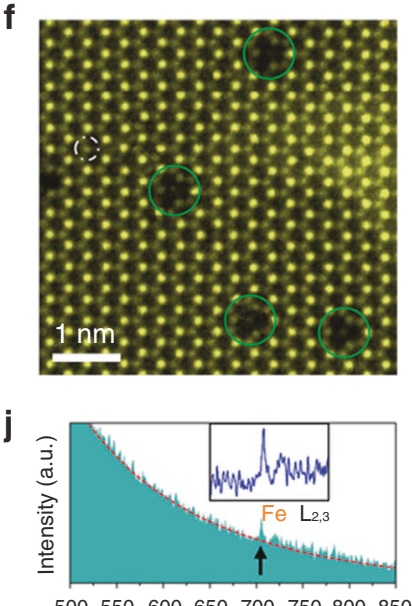

500550600650700750800850

Energy loss (eV)
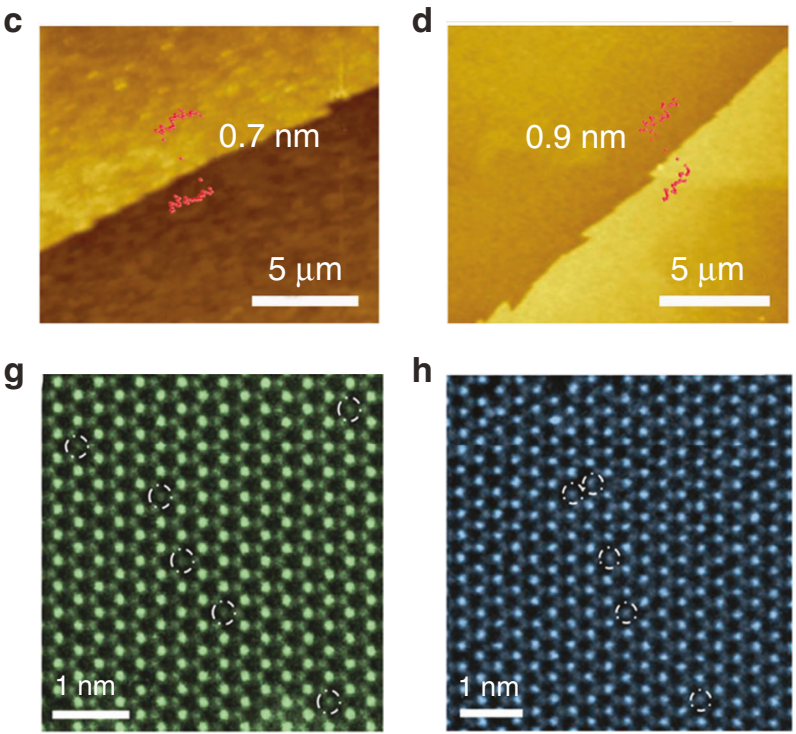

k

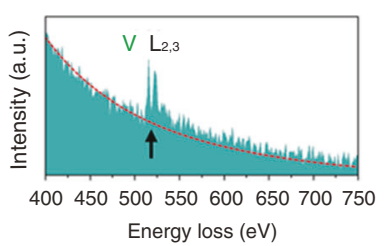

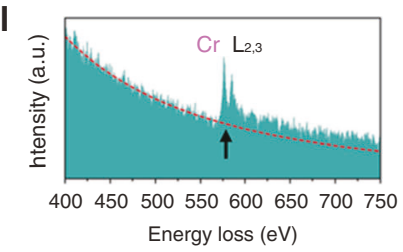

Fig. 2 Imaging of $\mathbf{M o S}_{\mathbf{2}}$ monolayers with various local configurations. AFM images of a $3 \mathrm{Co}_{M o}-V_{s}, \mathbf{b} 3 \mathrm{Fe}_{\mathrm{Mo}}-\mathrm{Vs}_{\mathrm{s}} \mathbf{c} 1 \mathrm{~V}_{\mathrm{Mo}}$, and $\mathbf{d} 1 \mathrm{Cr} \mathrm{Mo}_{\mathrm{ompl}}$ sam, illustrating the monolayer nature of the as-synthesized $\mathrm{MoS}_{2}$. e-h Atomic resolution STEM images of the TM-containing MoS 2 samples with i-I corresponding electron energy loss spectrum on single TM. The images confirm that, while Co and Fe atoms prefer to form triangular clusters where three TM atoms connect a central sulfur vacancy $\left(3 \mathrm{TM}_{\mathrm{Mo}_{\mathrm{O}}}-V_{\mathrm{s}}\right.$, highlighted by green circles), $\mathrm{V}$ and $\mathrm{Cr}$ only form substitutional single atom without sulfur vacancy (1TM $\mathrm{Mo}_{\mathrm{\prime}}$ highlighted by white dashed circles), in good consistence with the theoretical prediction.

typical Mo atoms but similar to $S_{2}$ columns due to the nature of the STEM imaging ${ }^{27}$. The line intensity profile of the single isolated dopant site and comparison to the STEM simulation (Supplementary Fig. 16) confirm that the TM atoms successfully doped into the lattice rather than the adatoms on the surface of $\mathrm{MoS}_{2}$. A more careful inspection reveals two main types of local configurations as predicted by previous theoretical calculations: one TM atom substitutes the Mo site forming an isolated single TM site, as marked by the white circles in all four TM-containing $\mathrm{MoS}_{2}$ monolayers (Fig. 2g, h, k, l); three TM atoms forming a triangular cluster with a connecting central sulfur vacancy, named as $3 \mathrm{TM}_{\mathrm{Mo}}-V s$, as highlighted by green circles in $\mathrm{Co}$ and $\mathrm{Fe}-$ containing system (Fig. 2e, f, i, j). However, we find the $3 \mathrm{TM}_{\mathrm{Mo}}-V s$ are the dominating configuration in the Co- and Fe-containing $\mathrm{MoS}_{2}$ monolayers. The single isolated TM sites account for very small ratio $(<10 \%)$ compared to the $3 \mathrm{TM}_{\mathrm{Mo}}-V s$ (Supplementary Figs. 17, 20c, and 21c; Supplementary Notes 3, 6, and 7), which corroborates that the latter are the dominating causes towards the HER activity.

On the other hand, $\mathrm{Cr}$ and $\mathrm{V}$ form predominantly isolated single TM sites in the $\mathrm{MoS}_{2}$ lattice. This is due to the different formation energy of the two types of local configurations with different TM atoms. The single atom EELS measurements on the $\mathrm{TM}$ in each image further confirm the chemical identity of the corresponding introduced element, as recognized by the sharp L edges of $\mathrm{Co}, \mathrm{Fe}, \mathrm{V}$, and $\mathrm{Cr}$, respectively, offering strong evidence of the presence of TM atoms and the consistence of the predicted local configurations. The reference EELS spectra taken away from the dopant site (Supplementary Fig. 18 and Supplementary Note 4) confirms the observed sharp peaks in the spectra of Fig. 2 are not an artifact during the collection at the dopant site.
Electrochemical test of $\mathrm{MoS}_{2}$ with local configurations. To verify the predicted HER activity of in-plane sulfur modulated by designed local configurations, the effects of $3 \mathrm{Co}_{\mathrm{Mo}}-V s$, $3 \mathrm{Fe}_{\mathrm{Mo}}-V s, 1 \mathrm{Cr}_{\mathrm{Mo}}$, and $1 \mathrm{~V}_{\mathrm{Mo}}$ on $\mathrm{HER}$ catalytic activity are examined using a three-electrode electrochemical cell in an electrolyte containing $0.5 \mathrm{M} \mathrm{H}_{2} \mathrm{SO}_{4}$. Data are compared to pristine $\mathrm{MoS}_{2}$ and commercial Pt/C. Before LSV tests, electrochemical activation was implemented. Stable CVs of configured $\mathrm{MoS}_{2}$ samples after electrochemical activation process (black curves in Supplementary Fig. 19 and Supplementary Note 5) indicate no phase change during activation. Figure $3 a$ shows linear-sweep voltammograms (LSV) in the cathodic direction after the correction of ohmic potential drop (i.e., iR), where the currents are normalized to the electrode geometric area. It is seen that the pristine $\mathrm{MoS}_{2}$ with an overpotential of $317 \mathrm{mV}$ at $10 \mathrm{~mA} \mathrm{~cm}^{-2}\left(\eta_{10}\right)$ shows an inferior HER activity than those of configured $\mathrm{MoS}_{2}$. The optical image of pristine $\mathrm{MoS}_{2}$ as shown in Supplementary Fig. 22 shows the similar edge length with that of configured $\mathrm{MoS}_{2}$, excluding the edge effect on different activity. Both the $3 \mathrm{Fe}_{\mathrm{Mo}}-V s$ and the $1 \mathrm{Cr}_{\mathrm{Mo}}$ samples exhibit values of $\eta_{10}$ over $200 \mathrm{mV}$. On the contrary, the $3 \mathrm{Co}_{\mathrm{Mo}}-V s$ and the $1 \mathrm{~V}_{\mathrm{Mo}}$ show significantly reduced $\eta_{10}$ values down to below $150 \mathrm{mV}$. In particular, the $3 \mathrm{Co}_{\mathrm{Mo}}-V s$ has a lowest $\eta_{10}$ value of only $75 \mathrm{mV}$. The first cycles of $\mathrm{CV}$ for HER were operated to verify the structural stability of introduced basal configurations after activation (Supplementary Fig. 19). The trend of activity shown in the first CVs of configured $\mathrm{MoS}_{2}$ exhibits the same as that of LSV curves. Note that the Co atoms in $3 \mathrm{Co}_{\mathrm{Mo}}-V s$ configuration has an over $90 \%$ occupancy among the total Co atoms. Hence, even though a minor content $(<0.2$ at $\%)$ of 1 Co configuration is detected, we believe the $3 \mathrm{Co}_{\mathrm{Mo}}-V s$ configuration plays the 

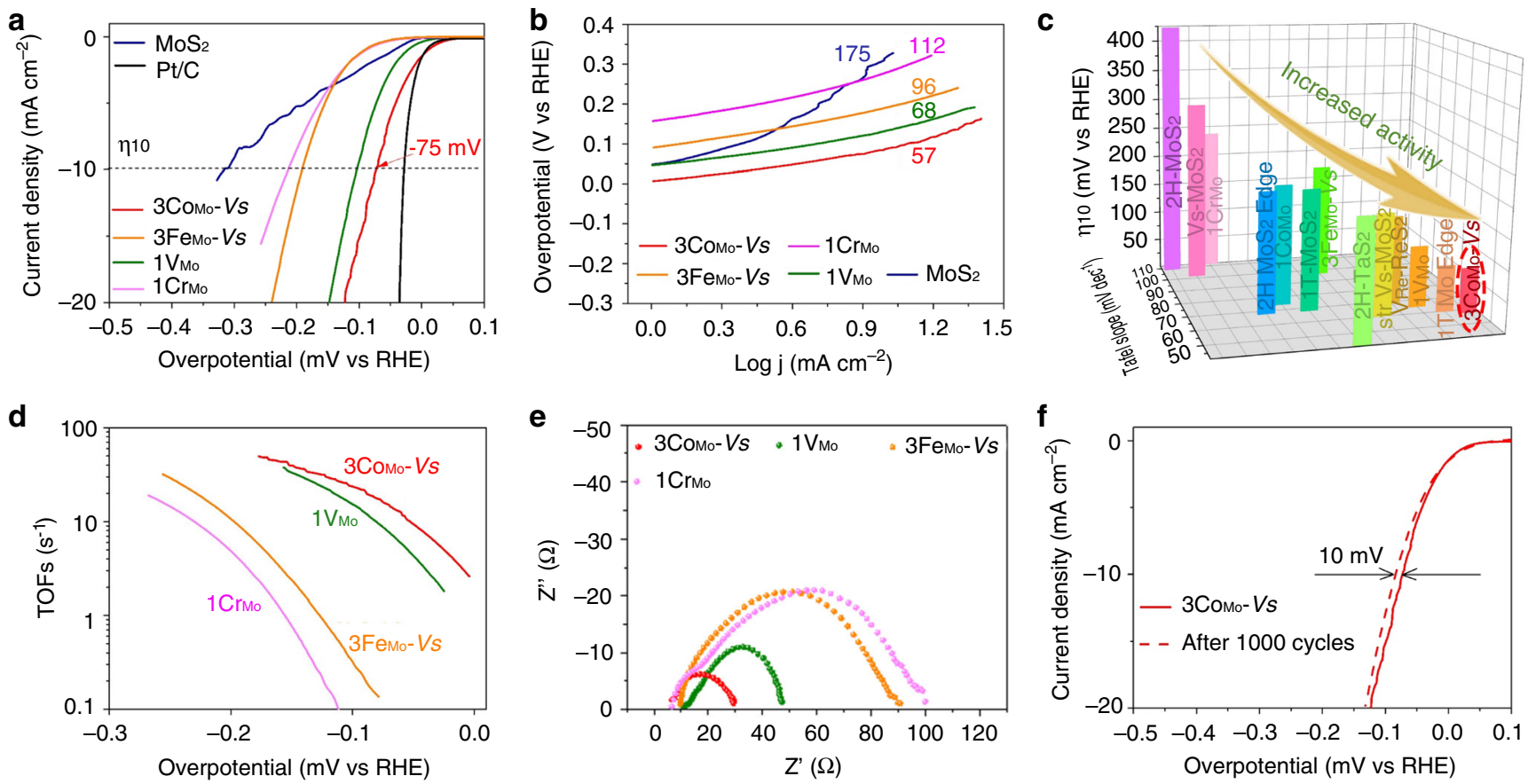

Fig. 3 HER properties of samples with different local configuration types. a Polarization curves of pristine $\mathrm{MoS}_{2}, \mathrm{MoS}_{2}$ with $3 \mathrm{Co}_{\mathrm{Mo}}-V_{\mathrm{s}}, 3 \mathrm{Fe} \mathrm{Mo}_{\mathrm{O}}-\mathrm{Vs}_{\mathrm{s}}, 1 \mathrm{~V}_{\mathrm{Mo}}$ $1 \mathrm{Cr}_{\mathrm{Mo}}$ configurations and $\mathrm{Pt} / \mathrm{C}$. The currents are normalized to the projected geometric area of the electrode. $\mathbf{b}$ The corresponding Tafel plots of the polarization curves in a. c Comparison of $\eta_{10}$-Tafel slope for $\mathrm{HER}$ catalysts in $0.5 \mathrm{M} \mathrm{H}_{2} \mathrm{SO}_{4}$. $\mathrm{MoS}_{2}$ with $3 \mathrm{Co}_{\mathrm{Mo}}-V s$ configuration exhibits top performance. The data are taken from refs. ${ }^{16,42-48}$. d TOFs of $\mathrm{MoS}_{2}$ with varied configuration types. e Electrochemical impedance spectroscopy (EIS) Nyquist plots for sample $3 \mathrm{Co}_{\mathrm{Mo}_{\mathrm{o}}}-\mathrm{Vs}_{\mathrm{s}}, 3 \mathrm{Fe}_{\mathrm{Mo}_{\mathrm{o}}}-\mathrm{Vs}_{\mathrm{s}} 1 \mathrm{~V}_{\mathrm{Mo}}$, and $1 \mathrm{Cr}_{\mathrm{Mo}_{\mathrm{o}}}$. $\mathbf{f}$ Long-test stability test for the $3 \mathrm{Co}_{\mathrm{Mo}_{\mathrm{o}}}-\mathrm{Vs}_{\mathrm{s}}$ electrode at a current density of $10 \mathrm{~mA} \mathrm{~cm}^{-2}$. .

dominating role in the catalyst activity. This is also in concise with the calculation that $\Delta G_{\mathrm{H}}$ of $3 \mathrm{Co}_{\mathrm{Mo}}-V s$ is closest to $0 \mathrm{eV}$ (Supplementary Table 1). The corresponding Tafel plots show the same trend with that of $\eta_{10}$ (Fig. $3 \mathrm{~b}$ ). The $3 \mathrm{Co}_{\mathrm{Mo}}-V s$ configuration sharply reduces the Tafel slope from $175 \mathrm{mV} \mathrm{dec}^{-1}$ in pristine $\mathrm{MoS}_{2}$ to $57 \mathrm{mV} \mathrm{dec}{ }^{-1}$. And the $1 \mathrm{~V}_{\mathrm{Mo}}$ sample gives an acceptable value of $68 \mathrm{mV} \mathrm{dec}{ }^{-1}$. In comparison, the $3 \mathrm{Fe}_{\mathrm{Mo}}-V s$ and $1 \mathrm{Cr}_{\mathrm{Mo}}$ configurations have little effect to the Tafel slope. Therefore, the lowered Tafel slopes of the $3 \mathrm{Co}_{\mathrm{Mo}}-V s$ and $1 \mathrm{~V}_{\mathrm{Mo}}$ with a fast discharge process of protons ${ }^{41}$ (Supplementary Note 8 ), may reflect a strengthened capability to adsorb $\mathrm{H}$. The Faradic efficiency was determined from the produced $\mathrm{H}_{2}$ characterized quantitatively by gas chromatography. As shown in Supplementary Fig. 23, the $3 \mathrm{Co}_{\mathrm{Mo}}-V s$ sample exhibits $>98 \%$ efficiency over the time scale of the measurement, confirming the $\mathrm{H}_{2}$ as the dominating product during the whole electrolysis process. From the overall comparison (Fig. 3c), we can conclude that the $3 \mathrm{Co}_{\mathrm{Mo}}-V s$ configuration, with synergistic triangular $\mathrm{Co}$ clusters surrounding one Vs in the center, renders $\mathrm{MoS}_{2}$ monolayer the best HER catalytic performance among all the configured $\mathrm{MoS}_{2}$ samples ${ }^{16,42-48}$. Supplementary Table 2 provides an extensive comparison to other TMDs and non-noble metal catalysts in their electrocatalysis of HER. The performance of our $\mathrm{MoS}_{2}$ monolayer with $3 \mathrm{Co}_{\mathrm{Mo}}-V s$ configuration exceeds all the pure TMD monolayer catalysts, and also compete with other non-noble metal catalysts.

The turnover frequency (TOF) per sulfur is calculated in order to correlate the intrinsic activity per sulfur atom with the local configuration (Supplementary Note 9). Each sulfur site tuned by $3 \mathrm{Co}_{\mathrm{Mo}}-V s$ or $1 \mathrm{~V}_{\mathrm{Mo}}$ possesses much higher efficiency than that in $3 \mathrm{Fe}_{\mathrm{Mo}}-V s$ and $1 \mathrm{Cr}_{\mathrm{Mo}}$ samples with increased value of TOFs. Compared to other configurations, the $3 \mathrm{Co}_{\mathrm{Mo}}-V s$ and $1 \mathrm{~V}_{\mathrm{Mo}}$ samples demonstrate the most appropriate tuning on the charge transfer capacity of local configuration.
Electrochemical impedance spectroscopy with fitted circuit models (Supplementary Fig. 24) shows significantly decreased charge-transfer resistances $\left(\mathrm{R}_{\mathrm{ct}}\right)$ for the $3 \mathrm{Co}_{\mathrm{Mo}}-V s(30.3 \Omega)$ and the $1 \mathrm{~V}_{\mathrm{Mo}}(47.9 \Omega)$ samples, as compared to those of $3 \mathrm{Fe}_{\mathrm{Mo}}-V s$ $(91.5 \Omega)$ and $1 \mathrm{Cr}_{\mathrm{Mo}}(100.3 \Omega)$, indicating a facilitated charge transfer between the $\mathrm{S}$ and protons in electrolyte (Fig. 3e). In addition, the $3 \mathrm{Co}_{\mathrm{Mo}}-V s$ sample exhibits an extraordinary longterm operation durability with small changes in potential (Fig. 3f and Supplementary Fig. 25). Hence, we may conclude that the $3 \mathrm{Co}_{\mathrm{Mo}}-V s$ configuration is efficient for HER during the whole cycling process.

Microcell measurements. In addition to the effect of local configuration type, it is expected that the concentration of such local configurations can also influence the amount of active sulfur sites. In order to prove the concentration effect, the on-chip electrochemical micro-devices are fabricated from a set of $3 \mathrm{Co}_{\mathrm{Mo}}-V s$ samples with local probe test, as shown in Fig. 4. Figure 4a, b show the three-electrode setup for the electrochemical measurements (more details are shown in Supplementary Fig. 26). In the first step, a controlled linear $I-V$ scan is done on the PMMA layer (Supplementary Fig. 27) to measure the electrochemical blocking reliability of the PMMA layer. The $\mathrm{MoS}_{2}$ samples with different $3 \mathrm{Co}_{\mathrm{Mo}}-V s$ concentrations (determined from STEM measurements, see Supplementary Fig. 21 and Supplementary Table 3) are applied for the microcell experiments. The obtained results are Fig. 4c-e. We can clearly see that an optimal Co concentration, corresponding to $\eta_{10}<100 \mathrm{mV}$, should be around 3.8 at $\%$, which translates to the $3 \mathrm{Co}_{\mathrm{Mo}}-V s$ concentration of $\sim 1.2$ at $\%$. The excessive increase in $3 \mathrm{Co}_{\mathrm{Mo}}-V s$ concentration results in performance decline. Possible reasons for this are deteriorated surface stability ${ }^{16}$ and superfluous lattice distortion ${ }^{18}$. This concentration effect also corroborates the key contribution of $3 \mathrm{Co}_{\mathrm{Mo}}-V s$ rather 
a
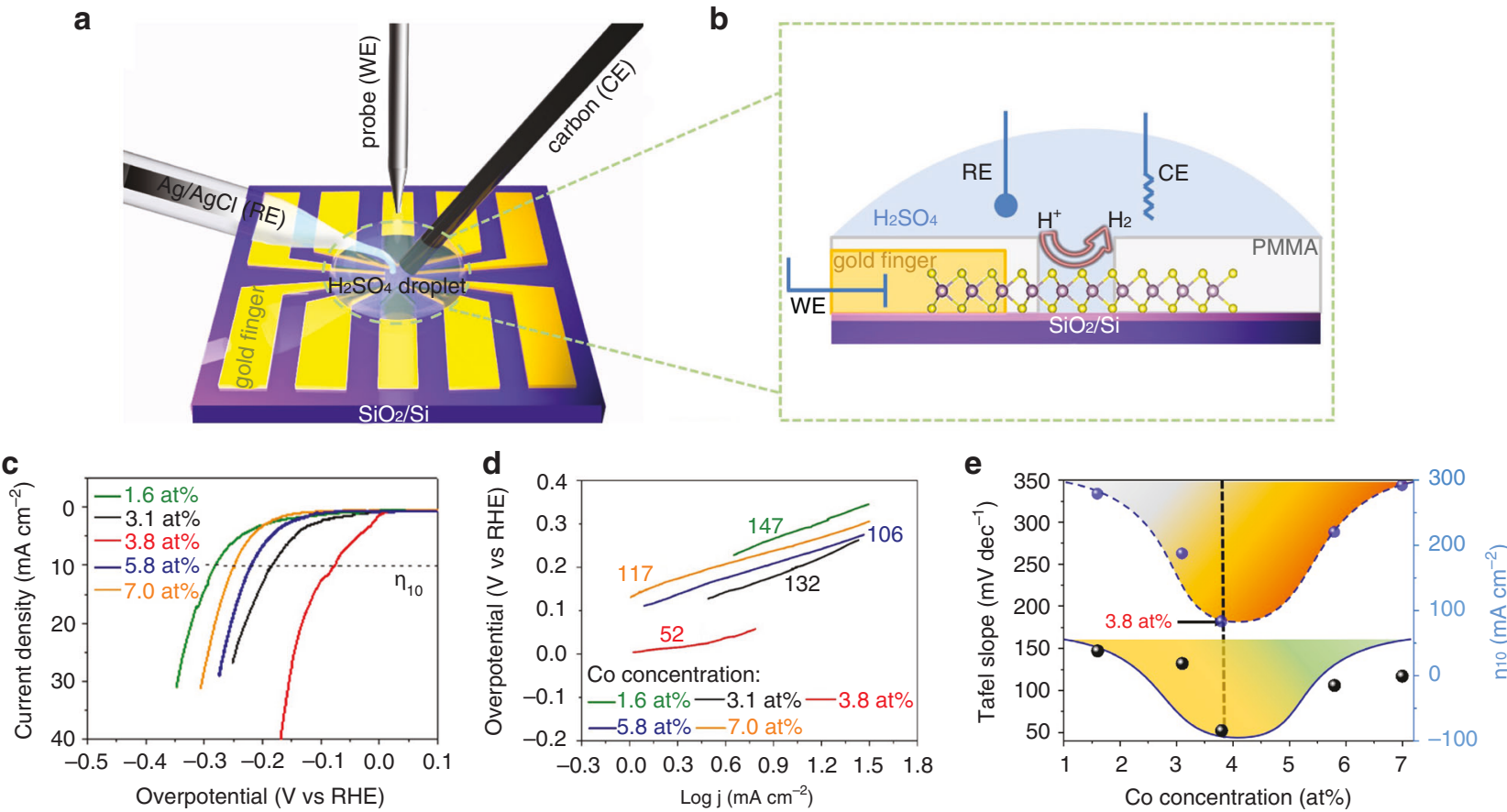

Fig. 4 Microcell HER measurements of the $\mathbf{3 C o}_{\mathbf{M o}}-V s$ samples. $\mathbf{a}, \mathbf{b}$ Schematic illustration of the microreactor device fabricated on $3 \mathrm{Co}_{\mathrm{Mo}}-V_{s}$ structured $\mathrm{MoS}_{2}$ monolayer. $\mathbf{c}$ LSV curves of the samples with Co concentrations of 1.6, 3.1, 3.8, 5.8, and 7.0 at\%. $\mathbf{d}$ The corresponding Tafel plots of the polarization curves in panel c. e The changing trends of Tafel slopes and $\eta_{10}$ with varied Co concentration. The optimal Co concentration is determined to be $\sim 3.8$ at $\%$, corresponding to the concentration of $3 \mathrm{Co}_{\mathrm{Mo}}-\mathrm{Vs}$ around 1.2 at $\%$.

than $1 \mathrm{Co}_{\mathrm{Mo}}$ to the HER catalyst activity enhancement. In addition, the microcell HER measurements are implemented on $3 \mathrm{Co}_{\mathrm{Mo}}-V s, 3 \mathrm{Fe}_{\mathrm{Mo}}-V s, 1 \mathrm{~V}_{\mathrm{Mo}}$, and $1 \mathrm{Cr}_{\mathrm{Mo}}$ samples with similar defect concentrations (Supplementary Fig. 28), showing the similar trend with that of three-electrode measurements (Fig. 3a).

\section{Discussion}

We have promoted the per-site electrochemical activity of inplane sulfur sites of $\mathrm{MoS}_{2}$ monolayer via tuning $\mathrm{H}-\mathrm{S}$ bonding strength, which can be understood by a hypothetical model of activating the inert sulfur atom into an open valence state. That activation can be correlated with the charge transfer capacity of local configuration. This is realized by forming various local configurations of transition metal atom or clusters $(\mathrm{Co}, \mathrm{Fe}, \mathrm{V}$, and $\mathrm{Cr})$ and compensative sulfur vacancy $(V s)$, which are confirmed by STEM images. In particular, the in-plane sulfur atoms modulated by $3 \mathrm{Co}_{\mathrm{Mo}}-V s$ configuration render the most active $\mathrm{MoS}_{2}$-based HER electrocatalyst in acidic medium to date (an overpotential $\eta_{10}$ of $75 \mathrm{mV}$ ). The optimized $3 \mathrm{Co}_{\mathrm{Mo}}-V s$ configuration is also verified by the systematic DFT calculations. In addition, the suitable Co concentration for the HER performance is achieved by the in-situ probe measurements of microcell. Our work highlights the potency of local configuration engineering in boosting the in-plane electrocatalytic activity of $\mathrm{MoS}_{2}$, as well as possibly other 2D TMD monolayers.

\section{Methods}

Synthesis of configured and pristine $\mathbf{M o S}_{\mathbf{2}}$. Pure $\mathrm{MoS}_{2}$ and configured $\mathrm{MoS}_{2}$ were synthesized by CVD method using $\mathrm{MoO}_{3}$ and sulfur (Sigma) as the precursor. For different TM-doped $\mathrm{MoS}_{2}, \mathrm{~V}_{2} \mathrm{O}_{5}, \mathrm{CrCl}_{3}, \mathrm{Fe}_{2} \mathrm{O}_{3}$, and $\mathrm{Co}_{3} \mathrm{O}_{4}$ were used as the corresponding TM sources. The synthesis was conducted using a quartz-tube single-zone furnace (1-inch diameter) in a temperature range from 550 to $650{ }^{\circ} \mathrm{C}$. Specifically, for the growth of pure $\mathrm{MoS}_{2}$, a quartz boat containing $10 \mathrm{mg} \mathrm{MoO}$ powder was put in the center of the tube, and the $\mathrm{SiO}_{2} / \mathrm{Si}$ substrate was placed on top of the quartz boat with the front side facing down. Another quartz boat containing $0.5 \mathrm{~g}$ sulfur powder was put upstream. The temperature ramped up to $700^{\circ} \mathrm{C}$ in $15 \mathrm{~min}$, and was maintained at the peak temperature for $5 \mathrm{~min}$ to $10 \mathrm{~min}$.
During the reaction, a constant $80 \mathrm{sccm}$ Ar flow was used as the carrier gas. After the reaction, the furnace cooled down naturally to room temperature. For the $3 \mathrm{Co}_{\mathrm{Mo}}-V s, 3 \mathrm{Fe}_{\mathrm{Mo}}-V s, 1 \mathrm{~V}_{\mathrm{Mo}}$, and $1 \mathrm{Cr}_{\mathrm{Mo}}$ structured $\mathrm{MoS}_{2}$, the precursor loaded in the central boat contained mixed powder of $\mathrm{V}_{2} \mathrm{O}_{5}, \mathrm{CrCl}_{3}, \mathrm{Fe}_{2} \mathrm{O}_{3}, \mathrm{Co}_{3} \mathrm{O}_{4}$, respectively, with $\mathrm{MoO}_{3}$ (mole ratio of 2: 98). The carrier gas used for the structured $\mathrm{MoS}_{2}$ was mixed $\mathrm{Ar} / \mathrm{H}_{2}$ with a flow of $80 / 5 \mathrm{sccm}$. The rest reaction conditions were the same as that for pure $\mathrm{MoS}_{2}$.

Structural characterizations. Room temperature Raman measurements were performed using a WITEC alpha $300 \mathrm{R}$ Confocal Raman system with an excitation laser of $532 \mathrm{~nm}$. The Raman system was pre-calibrated based on the Raman peak of crystalline $\mathrm{Si}$ at $520 \mathrm{~cm}^{-1}$. The laser power was kept below $1 \mathrm{~mW}$ to avoid sample heating. The TEM samples of the $3 \mathrm{Co}_{\mathrm{Mo}}-V s, 3 \mathrm{Fe}_{\mathrm{Mo}}-V s, 1 \mathrm{~V}_{\mathrm{Mo}}$, and $1 \mathrm{Cr}_{\mathrm{Mo}}$ structured $\mathrm{MoS}_{2}$ were prepared as follows. A layer of poly (methyl methacrylate) (PMMA) was spin-coated on the sample surface with a thickness of $\sim 1 \mu \mathrm{m}$, and then baked in an electric oven at $180^{\circ} \mathrm{C}$ for $3 \mathrm{~min}$. Afterwards, the substrates were immersed in a $\mathrm{NaOH}$ solution $(1 \mathrm{M})$ overnight to dissolve the $\mathrm{SiO}_{2}$ layer. After liftoff, the $\mathrm{MoS}_{2}$ samples were washed with DI water for several cycles. Then the monolayer samples were fished by a TEM grid (Quantifoil Mo grid). The obtained TEM specimen were dried naturally in ambient environment, and then dipped into high-purity acetone overnight to remove the PMMA layers. The STEM investigation was performed at room temperature on an aberration-corrected Nion UltraSTEM-100 and a JEOL $2100 \mathrm{~F}$ with a cold field-emission gun and an aberration corrector (the DELTA-corrector), both operating at $60 \mathrm{kV}$.

Electrochemical measurements. PMMA methylbenzene was uniformly spun on the $\mathrm{SiO}_{2} / \mathrm{Si}$ substrates deposited with monolayer $\mathrm{MoS}_{2}$. After baking at $100^{\circ} \mathrm{C}$ for 5 min, the PMMA film covered substrates were immersed in a $5 \mathrm{M} \mathrm{KOH}$ solution. As a result of the etching effect by $\mathrm{KOH}$, the monolayer $\mathrm{MoS}_{2}$ samples with the PMMA film were detached from the $\mathrm{SiO}_{2} / \mathrm{Si}$ substrate. Then, the obtained monolayer $\mathrm{MoS}_{2} / \mathrm{PMMA}$ films were washed in DI water and overlaid on the glassy carbon rotating disk electrode (RDE). After the thorough evaporation of DI water between the RDE electrode and the $\mathrm{MoS}_{2}$ /PMMA films, the PMMA films were further removed by dipping into acetone. As a result, the glassy carbon RDE electrode covered by monolayer $\mathrm{MoS}_{2}$ were obtained ${ }^{45,46}$.

For the electrochemical measurements, a standard three-electrode cell consisting of the glassy carbon RDE as the working electrode, a graphite carbon counter electrode and a saturated calomel reference electrode (SCE) was used. The electrolyte solution was $0.5 \mathrm{M} \mathrm{H}_{2} \mathrm{SO}_{4}$. An electrochemical workstation (CHI760) coupled with a RDE system (AFMSRCE3529, Pine Research Instrumentation, USA) was used to control the cell. The potential versus the reversible hydrogen electrode (RHE) was calculated according to $E_{\mathrm{RHE}}=E_{\mathrm{SCE}}+E_{\mathrm{SCE}}^{\mathrm{o}}(0.2412)+$ $0.059 \times \mathrm{pH}$. Before HER test, the catalysts went through an electrochemical 
activation process by cyclic voltammetry scanning in the same electrolyte $(0.5 \mathrm{M}$ $\mathrm{H}_{2} \mathrm{SO}_{4}$ ) with a scan rate of $100 \mathrm{mV} \mathrm{s}^{-1}$ in the potential range of 0.1 to $-0.29 \mathrm{~V}$ (vs. RHE). Linear sweep voltammetry (LSV) measurements were conducted with a scan rate of $2 \mathrm{mV} \mathrm{s}^{-1}$ under $1500 \mathrm{rpm}$. The current vs. potential plots were corrected by $90 \%$ ohmic compensation. The electrochemical impedance spectroscopy (EIS) were obtained in the same three-electrode configuration in the frequency range of $100 \mathrm{KHz}$ to $0.1 \mathrm{~Hz}$ and at an applied current of $10 \mathrm{~mA} \mathrm{~cm}^{-2}$. For the stability assessment, polarization data were measured in the beginning and after $1000 \mathrm{CV}$ sweeps $\left(-0.2\right.$ and $+0.2 \mathrm{~V}$ vs. RHE, scan rate: $\left.50 \mathrm{mV} \mathrm{s}^{-1}\right)$. In addition, the constantcurrent $\left(10 \mathrm{~mA} \mathrm{~cm}^{-2}\right)$ measurements were also implemented to evaluate the stability of potential.

The on-chip electrochemical measurements were carried out following the previous report ${ }^{11}$. Briefly, structured- $\mathrm{MoS}_{2}$ monolayers were transferred onto $\mathrm{SiO}_{2}$ $(300 \mathrm{~nm}) /$ Si substrate with pre-made gold fingers by PMMA assisted wet transfer method. Monolayers were further patterned into domains by e-beam lithography and 5-s treatment in nitrogen plasma. Contacts between gold fingers and monolayers were made via e-beam lithography and gold deposition processes. Microcell reaction windows were made by e-beam lithography on $1-\mu \mathrm{m}$-thick spincoated PMMA layer. During measurements, gold fingers connecting configured $\mathrm{MoS}_{2}$ monolayers, $\mathrm{Ag} / \mathrm{AgCl}$ encapsulated by Luggin capillary and carbon rod were used as working, reference and counter electrodes respectively. In all, $5 \mu \mathrm{l}$ of $0.5 \mathrm{M}$ $\mathrm{H}_{2} \mathrm{SO}_{4}$ (degassed with Ar bubbling for $10 \mathrm{~min}$ ) was used as electrolyte for each test. The scan rate for the LSV tests were $10 \mathrm{mV} / \mathrm{s}$.

Computational methods. The density functional theory (DFT) calculations were perfomed using the Vienna Ab initio simulation package ${ }^{49,50}$. The generalized gradient approximation with the Perdew-Burke-Ernzerhof exchange-correlation fuctional and a $450-\mathrm{eV}$ cutoff for the plane-wave basis set are employed ${ }^{51}$. The projector-augmented plane wave was adopted to describe the electron-ion interactions $^{52}$. The em piracal dispersions of Grimme (DFT-D2) was applied to account for the long-range van der Waals interacions ${ }^{53}$. All calculations were spin-polarized and the convergence threshold was set to be $10^{-4} \mathrm{eV}$ in energy and $0.01 \mathrm{eV} / \AA$. The k-point sampling of the Brillouin zone was obtained using a $4 \times 4 \times 1$ by the Monkhorst-Pack scheme. In addition, a $5 \times 5 \times 1$ supercell was also used to confirm the sufficiency of $4 \times 4 \times 1$ supercell (Supplementary Fig. 29). In the electronic structure calculation, denser k-points $(8 \times 8 \times 1)$ were used for better accuracy. The vacuum slab of $15 \AA$ was inserted in the z-direction for suface isolation to eliminate periodic interaction. The free energy of the adorbed state was calculated as

$$
\Delta G=\Delta E_{\mathrm{H}^{*}}+\Delta E_{\mathrm{ZPE}}-T \Delta S,
$$

where $\Delta E_{\mathrm{H}^{*}}$ is the hydrogen chemisorption energy, and $\Delta E_{\mathrm{ZPE}}$ is the difference of the zero point energy between the adsorbed state and the gas phase. Considering the fact the vibriation entropy of $\mathrm{H}^{*}$ in the adsorbed state is very small, the entropy of $1 / 2 \mathrm{H}_{2}$ adsorption can be approximated as $\Delta S_{\mathrm{H}} \approx-1 / 2 S_{\mathrm{H}^{2}}^{0}$, where $S_{\mathrm{H}^{2}}^{0}$ is the entropy of $\mathrm{H}_{2}$ in the gas phase at the standard conditions.

\section{Data availability}

All relevant data are available from the authors.

Received: 5 March 2019; Accepted: 15 April 2020;

Published online: 07 May 2020

\section{References}

1. Hinnemann, B. et al. Biornimetic hydrogen evolution: $\mathrm{MoS}_{2}$ nanoparticles as catalyst for hydrogen evolution. J. Am. Chem. Soc. 127, 5308-5309 (2005).

2. Kibsgaard, J., Jaramillo, T. F. \& Besenbacher, F. Building an appropriate active-site motif into a hydrogen-evolution catalyst with thiomolybdate $\left[\mathrm{Mo}_{3} \mathrm{~S}_{13}\right]^{2-}$ clusters. Nat. Chem. 6, 248-253 (2014).

3. Shi, J. P. et al. Controllable growth and gransfer of mono layer $\mathrm{MoS}_{2}$ on $\mathrm{Au}$ foils and its potential application in hydrogen evolution reaction. ACS Nano $\mathbf{8}$, 10196-10204 (2014)

4. Li, Y. et al. $\mathrm{MoS}_{2}$ nanoparticles grown on graphene: an advanced catalyst for the hydrogen evolution reaction. J. Am. Chem. Soc. 133, 7296-7299 (2011).

5. Tang, Y.-J. et al. Molybdenum disulfide/nitrogen-doped reduced graphene oxide nanocomposite with enlarged interlayer spacing for electrocatalytic hydrogen evolution. Adv. Energy Mater. 6, 1600116 (2016).

6. Li, D. J. et al. Molybdenum sulfide/N-doped CNT forest hybrid catalysts for high-performance hydrogen evolution reaction. Nano Lett. 14, 1228-1233 (2014).

7. Luo, Z. Y. et al. Chemically activating $\mathrm{MoS}_{2}$ via spontaneous atomic palladium interfacial doping towards efficient hydrogen evolution. Nat. Commun. $\mathbf{9}$, 2120 (2018).
8. Tsai, C., Abild-Pedersen, F. \& Norskov, J. K. Tuning the $\mathrm{MoS}_{2}$ edge-site activity for hydrogen evolution via support interactions. Nano Lett. 14, 1381-1387 (2014)

9. Ye, G. L. et al. Defects engineered monolayer $\mathrm{MoS}_{2}$ for improved hydrogen evolution reaction. Nano Lett. 16, 1097-1103 (2016).

10. Xie, J. F. et al. Defect-rich $\mathrm{MoS}_{2}$ ultrathin nanosheets with additional active edge sites for enhanced electrocatalytic hydrogen evolution. Adv. Mater. 25 5807-5813 (2013).

11. Zhang, J. et al. Unveiling active sites for the hydrogen evolution reaction on monolayer $\mathrm{MoS}_{2}$. Adv. Mater. 29, 1701955 (2017).

12. Kan, M. et al. Structures and phase transition of a $\mathrm{MoS}_{2}$ monolayer. J. Phys. Chem. C 118, 1515-1522 (2014).

13. Lukowski, M. A. et al. Enhanced hydrogen evolution catalysis from chemically exfoliated metallic $\mathrm{MoS}_{2}$ nanosheets. J. Am. Chem. Soc. 135, 10274-10277 (2013).

14. Chang, Y. H. et al. Highly efficient electrocatalytic hydrogen production by $M_{0} S_{x}$ grown on graphene-protected 3D Ni foams. Adv. Mater. 25, 756-760 (2013).

15. Tran, P. D. et al. Coordination polymer structure and revisited hydrogen evolution catalytic mechanism for amorphous molybdenum sulfide. Nat. Mater. 15, 640-646 (2016).

16. Li, H. et al. Activating and optimizing $\mathrm{MoS}_{2}$ basal planes for hydrogen evolution through the formation of strained sulphur vacancies. Nat. Mater. 15, 48-53 (2016)

17. Ouyang, Y. X. et al. Activating inert basal planes of $\mathrm{MoS}_{2}$ for hydrogen evolution reaction through the formation of different intrinsic defects. Chem. Mater. 28, 4390-4396 (2016).

18. Deng, J. et al. Multiscale structural and electronic control of molybdenum disulfide foam for highly efficient hydrogen production. Nat. Commun. 8, 14430 (2017).

19. Sun, T. et al. Defect chemistry in 2D materials for electrocatalysis. Mater. Today Energy 12, 215-238 (2019).

20. Alarawi, A., Ramalingam, V. \& He, J.-H. Recent advances in emerging single atom confined two-dimensional materials for water splitting applications. Mater. Today Energy 11, 1-23 (2019).

21. Li, H. L. et al. Synergetic interaction between neighbouring platinum monomers in $\mathrm{CO}_{2}$ hydrogenation. Nat. Nanotechnol. 13, 411-417 (2018).

22. Suh, J. et al. Reconfiguring crystal and electronic structures of $\mathrm{MoS}_{2}$ by substitutional doping. Nat. Commun. 9, 199 (2018).

23. Cho, K. et al. Electrical and optical characterization of $\mathrm{MoS}_{2}$ with sulfur vacancy passivation by treatment with alkanethiol molecules. ACS Nano 9 8044-8053 (2015)

24. Li, H. et al. Kinetic study of hydrogen evolution reaction over strained $\mathrm{MoS}_{2}$ with sulfur vacancies using scanning electrochemical microscopy. J. Am. Chem. Soc. 138, 5123-5129 (2016).

25. Enyashin, A. N. et al. New route for stabilization of $1 \mathrm{~T}-\mathrm{WS}_{2}$ and $\mathrm{MoS}_{2}$ phases J. Phys. Chem. C 115, 24586-24591 (2011).

26. Liu, P. X. et al. Photochemical route for synthesizing atomically dispersed palladium catalysts. Science 352, 797-801 (2016).

27. Zhou, W. et al. Intrinsic structural defects in monolayer molybdenum disulfide. Nano Lett. 13, 2615-2622 (2013).

28. Hong, J. H. et al. Exploring atomic defects in molybdenum disulphide monolayers. Nat. Commun. 6, 6293 (2015).

29. $\mathrm{Yu}, \mathrm{Z}$. H. et al. Towards intrinsic charge transport in monolayer molybdenum disulfide by defect and interface engineering. Nat. Commun. 5, 5290 (2014).

30. Zhu, C. R., Gao, D., Ding, J., Chao, D. \& Wang, J. TMD-based highly efficient electrocatalysts developed by combined computational and experimental approaches. Chem. Soc. Rev. 47, 4332-4356 (2018).

31. Li, L. \& Carter, E. A. Defect-mediated charge-carrier trapping and nonradiative recombination in $\mathrm{WSe}_{2}$ monolayers. J. Am. Chem. Soc. 141, 10451-10461 (2019)

32. Li, L., Long, R., Bertolini, T. \& Prezhdo, O. V. Sulfur adatom and vacancy accelerate charge recombination in $\mathrm{MoS}_{2}$ but by different mechanisms: timedomain ab initio analysis. Nano Lett. 17, $7962-7967$ (2017).

33. Lee, Y. L., Kleis, J., Rossmeisl, J., Shao-Horn, Y. \& Morgan, D. Prediction of solid oxide fuel cell cathode activity with first-principles descriptors. Energy Environ. Sci. 4, 3966-3970 (2011).

34. Cai, L. et al. Vacancy-induced ferromagnetism of $\mathrm{MoS}_{2}$ nanosheets. J. Am. Chem. Soc. 137, 2622-2627 (2015).

35. Sim, D. et al. Controlled doping of vacancy-containing few-layer $\mathrm{MoS}_{2}$ via highly stable thiol-based molecular chemisorption. ACS Nano 9, 12115-12123 (2015).

36. Nguyen, E. P. et al. Electronic Tuning of $2 \mathrm{D} \mathrm{MoS}_{2}$ through Surface Functionalization. Adv. Mater. 27, 6225-6229 (2015).

37. Greeley, J. \& Mavrikakis, M. Alloy catalysts designed from first principles. Nat. Mater. 3, 810-815 (2004). 
38. Greeley, J., Jaramillo, T., Bonde, J., Chorkendorff, I. \& Norskov, J. K. Computational high-throughput screening of electrocatalytic materials for hydrogen evolution. Nat. Mater. 5, 909-913 (2006).

39. Li, H. et al. From bulk to monolayer $\mathrm{MoS}_{2}$ : evolution of raman scattering. $A d v$. Funct. Mater. 22, 1385-1390 (2012).

40. Tietze, M. L., Burtone, L., Riede, M., Lüssem, B. \& Leo, K. Fermi level shift and doping efficiency inp-doped small molecule organic semiconductors: a photoelectron spectroscopy and theoretical study. Phys. Rev. B 86, 035320 (2012).

41. Downes, C. A. \& Marinescu, S. C. Bioinspired metal selenolate polymers with tunable mechanistic pathways for efficient $\mathrm{H}_{2}$ evolution. ACS Catal. 7, 848-854 (2017).

42. Yin, Y. et al. Contributions of phase, sulfur vacancies, and edges to the hydrogen evolution reaction catalytic activity of porous molybdenum disulfide nanosheets. J. Am. Chem. Soc. 138, 7965-7972 (2016).

43. Li, G. et al. All the catalytic active sites of $\mathrm{MoS}_{2}$ for hydrogen evolution. J. Am. Chem. Soc. 138, 16632-16638 (2016)

44. Yilmaz, G. et al. In situ transformation of MOFs into layered double hydroxide embedded metal sulfides for improved electrocatalytic and supercapacitive performance. Adv. Mater. 29, 1606814 (2017).

45. Geng, X. M. et al. Pure and stable metallic phase molybdenum disulfide nanosheets for hydrogen evolution reaction. Nat. Commun. 7, 10672 (2016).

46. Shi, J. P. et al. Two-dimensional metallic tantalum disulfide as a hydrogen evolution catalyst. Nat. Commun. 8, 958 (2017).

47. Zhou, Y. et al. Auto-optimizing hydrogen evolution catalytic activity of $\mathrm{ReS}_{2}$ through intrinsic charge engineering. ACS Nano 12, 4486-4493 (2018).

48. Voiry, D. et al. The role of electronic coupling between substrate and 2D $\mathrm{MoS}_{2}$ nanosheets in electrocatalytic production of hydrogen. Nat. Mater. 15, 1003-1009 (2016).

49. Kresse, G. \& Furthmüller, J. Efficiency of ab-initio total energy calculations for metals and semiconductors using a plane-wave basis set. Comput. Mater. Sci. 6, 15-50 (1996).

50. Kresse, G. \& Furthmuller, J. Efficient iterative schemes for ab initio totalenergy calculations using a plane-wave basis set. Phys. Rev. B 54, 11169-11186 (1996).

51. Fang, Y. et al. Structural determination and nonlinear optical properties of new $1 T^{\prime \prime \prime}$-Type $\mathrm{MoS}_{2}$ compound. J. Am. Chem. Soc. 141, 790-793 (2019).

52. Blochl, P. Projector augmented-wave method. Phys. Rev. B 50, 17953-17979 (1994).

53. Grimme, S. Semiempirical GGA-type density functional constructed with a long-range dispersion correction. J. Comput. Chem. 27, 1787-1799 (2006).

\section{Acknowledgements}

H.J.F. and Y.Z. thank the financial support by Singapore MOE under its Tier 2 grant (MOE2017-T2-1-073). Y.Z. appreciates the financial support from the Shanghai Natural Science Foundation of China (19ZR1465100). E.H.S. and J.J.L. thank the financial support from National Natural Science Foundation $(21973107,51702345)$. J.D.Z. and Z.L. thank the financial support from MOE Tier 2 grant MOE2016-T2-1-131. J.H.L. thanks the support from National Natural Science Foundation of China (11974156) and Guandong International Science Collaboration Project (Grant No. 2019A050510001).
W.Z. thanks the financial support from National Key R\&D Program of China (2018YFA035800). K.S. acknowledges JST-ACCEL and JSPS KAKENHI (JP16H06333, JP25107003, and P16382) for financial support. J.Z. and J.L. appreciate the financial support from Welch Foundation C-1716 and the NSF I/UCRC Center for Atomically Thin Multifunctional Coatings (ATOMIC) under award \# IIP-1539999. We appreciate the supports of X.G.Li and S.S. Tang from Nanyang Technological University on the measurements of Faradic efficiency.

\section{Author contributions}

Y.Z., Z.L., J.L., and H.J.F. conceived the project. Y.Z., J.Z., and J.D.Z. designed the experiments. J.H. L.,W.Z., and K.S. performed the STEM characterization of the samples and data analysis. E.H.S. and J.J.L. proposed the local configuration electroneagtivity and carried out the theoretical calculations. Y.Z., J.Z., E.H.S. and J.J.L. wrote the paper with contributions from other co-authors. All authors discussed and commented on the manuscript.

\section{Competing interests}

The authors declare no competing interests.

\section{Additional information}

Supplementary information is available for this paper at https://doi.org/10.1038/s41467 020-16111-0.

Correspondence and requests for materials should be addressed to J.L., J.L. or H.J.F.

Peer review information Nature Communications thanks Ana Belén Muñoz-García and other, anonymous, reviewers for their contributions to the peer review of this work. Peer review reports are available.

Reprints and permission information is available at http://www.nature.com/reprints

Publisher's note Springer Nature remains neutral with regard to jurisdictional claims in published maps and institutional affiliations.

(c) (i) Open Access This article is licensed under a Creative Commons Attribution 4.0 International License, which permits use, sharing, adaptation, distribution and reproduction in any medium or format, as long as you give appropriate credit to the original author(s) and the source, provide a link to the Creative Commons license, and indicate if changes were made. The images or other third party material in this article are included in the article's Creative Commons license, unless indicated otherwise in a credit line to the material. If material is not included in the article's Creative Commons license and your intended use is not permitted by statutory regulation or exceeds the permitted use, you will need to obtain permission directly from the copyright holder. To view a copy of this license, visit http://creativecommons.org/ licenses/by/4.0/.

(C) The Author(s) 2020 